\title{
Ninjinyoeito Ameliorated Cigarette Smoke Extract- induced Apoptosis and Inflammation Through JNK Signaling Inhibition in Human Lung Fibroblasts
}

Kenta Murata ( $\square$ murata_kenta@kracie.co.jp )

Kracie (Japan)

Nina Fujita

Kracie (Japan)

Ryuji Takahashi

Kracie (Japan)

\section{Research Article}

Keywords: Ninjinyoeito, JNK, apoptosis, cigarette smoke

Posted Date: October 27th, 2021

DOl: https://doi.org/10.21203/rs.3.rs-941651/v1

License: (c) (i) This work is licensed under a Creative Commons Attribution 4.0 International License.

Read Full License

Version of Record: A version of this preprint was published at BMC Complementary Medicine and Therapies on March 31st, 2022. See the published version at https://doi.org/10.1186/s12906-022-035745 . 


\section{Abstract}

\section{Background}

Cigarette smoke is a major risk factor for various lung diseases, such as chronic obstructive pulmonary disease (COPD). Ninjinyoeito (NYT), a traditional Chinese medicine, has been prescribed for patients with post-illness or post-operative weakness, fatigue, loss of appetite, rash, cold limbs, and anemia. In addition to its traditional use, NYT has been prescribed for treating frailty in gastrointestinal, respiratory, and urinary functions. Further, NYT treatment can ameliorate cigarette smoke-induced lung injury, which is a destructive index in mice; however, the detailed underlying mechanism remains unknown.

\section{Purpose}

The purpose of this study was to investigate whether NYT ameliorates cigarette smoke-induced lung injury and inflammation in human lung fibroblasts and determine its mechanism of action.

\section{Methods}

We prepared a cigarette smoke extract (CSE) from commercially available cigarettes to induce cell injury and inflammation in the human lung fibroblast cell line HFL1. The cells were pretreated with NYT for $24 \mathrm{~h}$ prior to CSE exposure. Cytotoxicity and cell viability were measured by lactate dehydrogenase (LDH) cytotoxicity assay and cell counting kit (CCK)-8. IL-8 level in the cell culture medium was measured by performing Enzyme-Linked Immuno Sorbent Assay (ELISA). To clarify the mechanisms of NYT, we used CellROX Green Reagent for reactive oxygen species (ROS) production and western blotting analysis for cell signaling.

Results

Exposure of HFL1 cells to CSE for $24 \mathrm{~h}$ induced apoptosis and interleukin (IL)-8 release. Pretreatment with NYT inhibited apoptosis and IL-8 release. Furthermore, CSE exposure for $24 \mathrm{~h}$ increased the production of ROS and phosphorylation levels of p38 and JNK. Pretreatment with NYT only inhibited CSE-induced JNK phosphorylation, and not ROS production and p38 phosphorylation. These results suggest that NYT acts as a JNK-specific inhibitor.

\section{Conclusion}

NYT treatment ameliorated CSE-induced apoptosis and inflammation by inhibiting the JNK signaling pathway. Finally, these results suggest that NYT may be a promising therapeutic agent for patients with COPD.

\section{Introduction}


Cigarette smoke contains more than 7,000 chemicals, such as oxidative gases and heavy metals. It is well known that cigarette smoke is a major risk factor for various lung diseases, such as chronic obstructive pulmonary disease (COPD). Epidemiological and clinical studies have shown that smokers are significantly more likely to develop emphysema than nonsmokers, and the severity of the disease is directly correlated with the amount of cigarette smoke inhaled [1, 2]. Cigarette smoke causes direct oxidative stress damage on both cellular and extracellular structural elements of the lung, and initiates inflammatory processes that can cause indirect damage to the lungs. Cigarette smoke exposure to human lung fibroblasts has been reported to have various effects, such as apoptosis, cytokine release, and oxidative stress $[3,4]$. In particular, oxidative stress plays a central role in initiating and driving the mitogen-activated protein kinase (MAPK) signaling pathway, leading to various cellular responses following cigarette smoke exposure.

Ninjinyoeito (NYT), a traditional Chinese medicine consisting of 12 herbs, has been prescribed for patients with post-illness or post-operative weakness, fatigue, loss of appetite, cold limbs, and anemia. In addition to its traditional use, NYT has been recently prescribed for treating frailty in gastrointestinal, respiratory, and urinary functions [5]. In this context, it has been reported that NYT treatment for older patients with COPD can improve the COPD assessment test score, which is designed to measure the impact of COPD on a person's life and how it changes over time. In addition to the COPD assessment test score, NYT treatment also improves physical symptoms, such as body weight and muscle mass reduction, as well as psychological symptoms, such as depression and anxiety [6, 7]. In an in vivo study, NYT could ameliorate cigarette smoke-induced alveolar damage, as indicated by the destructive index in a mouse model of cigarette smoke-induced COPD. Although this previous report did not investigate the mechanism of action of NYT on lung damage, the authors suggested that NYT has some favorable effects on localized damage to the alveolar wall, including that on fibroblasts [8]. In addition, NYT has also been reported to have radical scavenging activity in hepatocytes [9] and can ameliorate nitric oxidemediated lung injury in a murine cytomegalovirus (MCMV)-infected model [10]. Therefore, we hypothesized that NYT possibly exerts a therapeutic effect against COPD-related scores through its antiinflammatory and anti-oxidant effects.

Thus, the purpose of this study was to clarify whether NYT ameliorated cigarette smoke-induced cytotoxicity and inflammation in human lung fibroblasts and to determine its mechanism of action.

\section{Materials And Methods}

Cell culture and treatment

Human male fetal lung fibroblast cells (HFL1) were purchased from the American Type Culture Collection (ATCC, Manassas, VA, USA). The cells were grown in Ham's F-12K medium (Thermo Fisher Scientific Ltd., Waltham, MA, USA) supplemented with $10 \%$ fetal bovine serum (FBS; Thermo Fisher Scientific Ltd.) and $1 \%$ antibiotics-antimycotics (Thermo Fisher Scientific Ltd.) in a humidified incubator at $37^{\circ} \mathrm{C}$ with $5 \% \mathrm{CO}_{2}$ as recommended by ATCC. Fibroblasts between 5 and 10 passages were used in all the experiments. 
Cigarette smoke extract preparation

Cigarette smoke extract (CSE) was prepared by bubbling smoke from two cigarettes (1.2 mg nicotine and $14 \mathrm{mg}$ tar) without a filter into $20 \mathrm{~mL}$ of serum-free Ham's F-12K medium at a rate of 1 cigarette/min as previously described [11]. The $\mathrm{pH}$ of the medium was adjusted to $7.4 \mathrm{using} \mathrm{HCl}$, and the medium was sterile filtered with a $0.22 \mu \mathrm{m}$ filter. The preparation was considered to be a $100 \%$ CSE concentration. CSE was then used in the subsequent experiments within $30 \mathrm{~min}$ of its preparation.

\subsection{Plant materials and extract preparation}

NYT is composed of 12 dried medicinal herbs described in Table 1, and was supplied by Kracie Pharma Ltd. (Tokyo, Japan) as a dried extract powder. Each plant material was identified by external morphology and authenticated by marker compounds of the plant specimens according to the method of Japanese Pharmacopeia and the standards of Kracie Pharma Ltd. NYT extract used in the study was industrially produced in Kracie Pharma Ltd according to the method of Japanese Pharmacopeia and the standards of Kracie Pharma Ltd. Purchasing manager purchased each plant material from various companies in China and the employee in Kracie Pharma Ltd. identified by comparing it with the standard specimens we own. Standard specimens are deposited in our laboratory, but we cannot deposit in a publicly available herbarium as it is confidential material. NYT extract (lot No. 15112017) was suspended in serum-free Ham's F-12K medium, and the medium was sterile filtered with a $0.22 \mu \mathrm{m}$ filter immediately before use. Also, the datasets used and/or analysed during the current study available from the corresponding author on reasonable request. The fingerprints of NYT was also performed as previously described [12].

\subsection{Cytotoxicity assay}

Cytotoxicity and cell viability were measured by lactate dehydrogenase (LDH) cytotoxicity assay (TaKaRa, Shiga, Japan) and cell counting kit (CCK)-8 (Dojindo Laboratories, Kumamoto, Japan), respectively, by following the manufacturer's instructions. The cells were treated with $2 \%$ Triton X-100 as a positive control for the LDH cytotoxicity assay. The cells were then seeded at a density of $1 \times 10^{4}$ cells/well in a 96-well cell culture plate in 10\% FBS Ham's F-12K medium. After $24 \mathrm{~h}$ of incubation, the cells were treated with different concentrations of NYT in 1\% FBS Ham's F-12K medium for $24 \mathrm{~h}$, and then $5 \%$ CSE with NYT was added. After $24 \mathrm{~h}$ of incubation, the cell culture plates were centrifuged at $250 \mathrm{~g}$ for $10 \mathrm{~min}$, and the supernatant was collected for performing the LDH assay. The LDH cytotoxicity assay kit was used to examine the level of LDH release, and the optical density of the samples was measured using a microplate reader Synergy H1 (BioTek, Tokyo, Japan) at $490 \mathrm{~nm}$. CCK-8 was used to examine the cell viability, and the optical density was measured using a microplate reader set at $450 \mathrm{~nm}$. To investigate the type of cell death that was induced by CSE exposure, the cells were treated with Z-VAD-FMK (Promega, Madison, WI, USA) or necrostatin-1 (Sellmeck Chemical, Houston, TX, USA) $2 \mathrm{~h}$ before the CSE exposure.

\subsection{Measurement of reactive oxygen species production}


The cells were seeded at a density of $1 \times 10^{4}$ cells/well in a 96-well cell culture plate in $10 \%$ FBS Ham's F$12 \mathrm{~K}$ medium. After $24 \mathrm{~h}$ of incubation, the cells were treated with different concentrations of NYT in $1 \%$ FBS Ham's F-12K medium for $24 \mathrm{~h}$, and then $5 \%$ CSE with NYT was added. After $8 \mathrm{~h}$ or $24 \mathrm{~h}$ of incubation, CellROX Green Reagent (Thermo Fisher Scientific Ltd.) was added to each well at a concentration of 10 $\mu \mathrm{mol} / \mathrm{L}$ for $30 \mathrm{~min}$. The fluorescence of CellROX was measured using the microplate reader Synergy H1.

\subsection{Western blotting analysis}

The cells were seeded at a density of $2 \times 10^{5}$ cells/well in a 6 -well cell culture plate in $10 \%$ FBS Ham's F$12 \mathrm{~K}$ medium. After $24 \mathrm{~h}$ of incubation, the cells were treated with $100 \mu \mathrm{g} / \mathrm{mL}$ NYT in $1 \%$ FBS Ham's F-12K medium for $24 \mathrm{~h}$, and then $5 \%$ CSE with NYT was added. After $24 \mathrm{~h}$ of incubation, the supernatants were collected for an Enzyme-linked ImmunoSorbent Assay, and the cells were collected using RIPA buffer (Fujifilm, Tokyo, Japan) supplemented with a protease-inhibitor cocktail (Nacalai Tesque, Kyoto, Japan) and a phosphatase-inhibitor cocktail (Nacalai Tesque). Western blot analysis was performed as previously described [13]. Lysates were centrifuged at $15,000 \mathrm{~g}$ for $20 \mathrm{~min}$ at $4^{\circ} \mathrm{C}$, and then $10 \mu \mathrm{g}$ aliquots of protein were separated on $10-20 \%$ SDS polyacrylamide gels, transferred onto polyvinylidene difluoride membranes (Merck Millipore, Burlington, MA, USA), and immunoblotted with the following primary antibodies at $4^{\circ} \mathrm{C}$ overnight: rabbit anti-cleaved caspase-3 monoclonal antibody (1:1000, Cell Signaling Technology [CST], Danvers, MA, USA), rabbit anti-cleaved PARP monoclonal antibody (1:1000,CST), rabbit anti-phospho-p44/42 MAPK (Erk1/2) monoclonal antibody (1:1000,CST), rabbit anti-phospho-p38 MAPK monoclonal antibody $(1: 1000, C S T)$, rabbit anti-phospho-SAPK/JNK monoclonal antibody $(1: 1000, C S T)$, rabbit anti-phospho-SEK1/MKK4 monoclonal antibody $(1: 1000, C S T)$, mouse anti- $\beta$-actin monoclonal antibody (1:1000; CST). The secondary antibodies used were HRP-conjugated goat anti-rabbit IgG (1:2000; CST) or goat anti-mouse IgG (1:5000; CST). Immunoreactive bands were visualized using an Amersham Imager 680 analyzer (GE Healthcare, Chicago, IL, USA). The band intensity was measured using Fiji software.

\subsection{IL-8 quantification in the medium by ELISA}

IL-8 level in the cell culture medium was measured by performing ELISA with human IL-8/CXCL8 (R\&D Systems, Minneapolis, MN, USA) by following the instructions in the product manual. Briefly, the collected cell culture medium was centrifuged at $250 \mathrm{~g}$ for $10 \mathrm{~min}$, and the supernatant was used as a sample.

\subsection{Statistical analysis}

All statistical analyses were performed using EZR (Saitama Medical Center, Jichi Medical University, Saitama, Japan), a graphical user interface for R (The R Foundation for Statistical Computing, Vienna, Austria). EZR is a modified version of $\mathrm{R}$ commander designed to add statistical functions frequently used in biostatistics. Statistical comparisons were performed using one-way analysis of variance followed by Tukey's test or Student's $t$-test. Differences were considered significant at $p<0.05$. All experiments were performed more than three times to confirm the reproducibility.

\section{Results}




\section{NYT inhibited CSE-induced cytotoxicity and IL-8 release}

We first investigated whether NYT treatment could inhibit CSE-induced cytotoxicity. The cells were treated with different concentrations of NYT for $24 \mathrm{~h}$ and then treated with $5 \%$ or $10 \%$ CSE preparation with NYT for $24 \mathrm{~h}$. As a result, treatment with both CSE concentrations showed a significant increase in LDH leakage in the medium than that in the control group; however, NYT pretreatment inhibited CSE-induced LDH leakage at both CSE concentrations in a dose-dependent manner (Figures 1A-B). In the CCK-8 cell viability assay, $5 \%$ CSE exposure enhanced and 10\% CSE exposure significantly inhibited mitochondrial activity at $24 \mathrm{~h}$ after exposure compared with that in the control group. In addition, NYT pretreatment did not alter the CSE-induced changes in mitochondrial activity (Figures 1C-D). In the subsequent experiments, we focused on the effect of NYT against $5 \%$ CSE-induced damage in HFL1 cells because CCK-8 values did not change after CSE exposure. Next, we investigated whether NYT treatment inhibited CSE-induced inflammation. We evaluated the content of IL-8 in the medium after CSE exposure. CSE exposure significantly increased IL-8 release into the medium $24 \mathrm{~h}$ after the exposure than that in the control group; however, NYT pretreatment significantly inhibited this release (Figure 2). These results suggest that NYT treatment inhibited CSE-induced cell damage and inflammation.

\section{NYT inhibited caspase-dependent apoptosis signal}

Next, we investigated the type of cell death that occurred in HFL1 cells exposed to $5 \%$ CSE. Two hours before CSE exposure, the HFL1 cells were treated with either Z-VAD-FMK or necrostatin-1 to inhibit apoptosis or necrosis, respectively. We observed that only Z-VAD-FMK treatment completely inhibited CSE-induced LDH leakage (Figures 3A-B). These results implied that CSE exposure to HFL1 cells mainly induced caspase-dependent apoptosis, and that NYT treatment might inhibit CSE-induced apoptosis. Since only Z-VAD-FMK treatment inhibited the CSE-induced LDH leakage, we evaluated the effects on cleaved-caspase 3 and cleaved PARP expression levels at $24 \mathrm{~h}$ after CSE treatment as a marker of apoptosis. CSE exposure increased the expression of both cleaved caspase 3 and cleaved PARP, and 100 $\mu \mathrm{g} / \mathrm{mL}$ NYT treatment significantly inhibited the increase in the expression of both the proteins $(\mathrm{p}=0.026$ or 0.047, respectively) (Figure 4). These results indicate that NYT treatment inhibited caspase-dependent apoptosis induced by CSE exposure.

\section{NYT did not inhibit CSE-induced reactive oxygen species production}

To clarify the mechanism of action of NYT on CSE-induced apoptosis, we investigated the effect of NYT on reactive oxygen species (ROS) production after CSE exposure. In a previous report, NYT treatment has shown a radical scavenging activity in rat hepatocytes and serum $[9,14]$. To measure the ROS production in living cells, the cells were treated with CellROX Green reagent for 30 min at $8 \mathrm{~h}$ or $24 \mathrm{~h}$ after the CSE treatment. Compared with the control group, the group with CSE exposure showed a significant increase in the ROS production; however, NYT treatment did not change the level of ROS production at either time point (Figure 5). These results indicated that the anti-oxidant activity of NYT was not involved in the antiapoptotic and anti-inflammatory effects of NYT. 
Next, we evaluated the MAPK signal activity after CSE treatment. There are three well-defined subgroups of MAPKs: extracellular signal-regulated kinases (ERK1/2), c-Jun $N$-terminal kinases (JNK), and p38 MAPKs. Without CSE exposure, NYT had no effect on the phosphorylation levels of p38, ERK1/2, and JNK compared with that in the control group. In contrast, $5 \%$ CSE exposure significantly increased the phosphorylation levels of p38 and JNK, but not of ERK1/2, at $24 \mathrm{~h}$ after the exposure compared with that in the control group. NYT pretreatment inhibited the phosphorylation of only JNK, and not of p38 (Figures 6A-D). JNKs are phosphorylated by the upstream dual specificity MAP2K, MKK4, or MKK7 molecules. CSE exposure significantly increased the phosphorylation level of MKK4; however, NYT treatment did not change the phosphorylation level (Figure 6E). These results suggest that NYT acts as a JNK-specific inhibitor.

\section{Discussion}

Here, we investigated whether NYT can ameliorate CSE-induced apoptosis and inflammation in the human lung fibroblast cell line HFL1 and further determined its mechanism of action. We observed that NYT inhibited caspase-dependent apoptosis and IL-8 release induced by CSE exposure. We also showed that NYT specifically inhibited the phosphorylation of JNK induced by CSE exposure. To our knowledge, this is the first study to reveal how NYT treatment ameliorates CSE-triggered apoptosis and inflammation in human lung fibroblasts.

NYT reportedly ameliorates cigarette smoke-induced alveolar damage as indicated by the destructive index; however, it does not affect the air space enlargement, as indicated by the mean linear intercept in a cigarette smoke-induced COPD mice model. This report suggests that NYT has some favorable effects on the localized damage to the alveolar wall, including that on fibroblasts, and that NYT has no effects on other connective structures, such as elastic fibers [8]. However, this report did not investigate the type of cells and the mechanism by which NYT treatment ameliorates cigarette smoke-induced lung injury. While cigarette smoke first targets the epithelium, which acts as a mechanical barrier and contributes to the development of the inflammatory response, water-soluble components of cigarette smoke can pass through the basement membrane and directly interact with the fibroblasts, which play a crucial role in maintaining the integrity of the alveolar structure. In the present study, we demonstrated that NYT treatment inhibited CSE-induced LDH leakage and IL-8 release. These results suggest that NYT has a protective effect against cigarette smoke-induced lung injury in the lung fibroblasts.

Oxidative stress plays a central role in initiating and driving signaling pathways that lead to cellular responses following CSE exposure. NYT reportedly inhibits tert-butyl hydroperoxide-induced damage via its radical scavenging activity in rat hepatocytes [9]. In addition, NYT suppresses the MCMV infectioninduced increase in iNOS and NO levels in serum [10]. Therefore, we hypothesized that NYT possibly suppresses CSE-induced apoptosis and inflammation via its anti-oxidant activity; however, NYT treatment 
did not change the CSE-induced ROS production at any time point. These results indicate that NYT has no radical scavenging activity and has no effect on anti-oxidant enzyme expression in HFL1.

Oxidative stress activates the MAPK family, which is a key component of the signaling pathways regulating various intracellular processes, including apoptosis and release of inflammatory cytokines [15]. As previously reported [16], CSE exposure significantly increases the phosphorylation levels of JNK and p38; in contrast, here, NYT only inhibited CSE-induced phosphorylation of JNK but not that of p38. JNKs are classic stress-activated protein kinases that are potently and preferentially activated after various cell stresses, including inflammatory cytokines, and growth factor withdrawal. In case of apoptosis, JNK is involved in both intrinsic and extrinsic apoptotic pathways. In the intrinsic apoptotic pathway, phosphorylated JNKs stimulate the expression of apoptosis-specific genes through target transcription factors, activate pro-apoptosis factors, such as BAX, and induce mitochondrial outer membrane permeabilization, leading to the release of cytochrome $\mathrm{c}$ from the mitochondria. The released cytochrome $\mathrm{c}$ forms apoptosomes, which trigger the caspase 9 cascade leading to the cleavage of caspase 3, caspase 6, and PARP, and consequently resulting in cellular apoptosis. In addition, JNK inhibits Bcl-2 activity, which is an anti-apoptotic protein, in both the direct and indirect pathways [17]. In contrast, the extrinsic pathway is characterized by apoptotic signaling initiated by the activation of death receptors by their respective ligands, such as TNF-a. Once the death receptor is activated, adaptor molecules, such as FAS-associated death domain protein, are recruited to the receptor complex, which activates caspase 8 [18]. Activated caspase 8 directly cleaves caspase 3 and caspase 7, leading to apoptosis. JNKs promote the phosphorylation and subsequent cleavage of Bid, leading to the inhibition of anti-apoptotic proteins such as XIAP and cIAP1, which are inhibitors of caspase 8. Inhibition of XIAP and CIAP1 leads to the activation of caspase 3 and caspase 7, ultimately leading to apoptosis. And a previous study has demonstrated that CSE induces apoptosis at lower concentrations (10-25\%) and necrosis at higher concentrations (50-100\%) in HFL1 cells, and that low concentrations of CSE exposure on HFL1 cells induces apoptosis more than it induces necrosis $[19,20]$. In the present study, LDH leakage after exposure to 5\% CSE was completely inhibited by Z-VAD-FMK treatment, a caspase inhibitor, and not necrostatin-1, a necrosis inhibitor. In addition, NYT treatment inhibited the cleavage of both caspase 3 and PARP at $24 \mathrm{~h}$ after CSE exposure. These results suggest that NYT treatment might inhibit CSEinduced activation of intrinsic and/or extrinsic apoptosis pathways through the inhibition of JNK activation.

JNKs are phosphorylated by the upstream dual specificity molecules MAPKK, MKK4, or MKK7; however, NYT did not change the phosphorylation level of MKK4. These results suggest that NYT treatment may activate JNK phosphatase. Among the phosphatases of MAPK, dual specificity phosphatase 16 (DUSP16) is a JNK-specific phosphatase [21, 22]. The forced expression of DUSP16 in COS-7 cells showed suppression of MAPK signal activation, especially that of JNK phosphorylation. In addition, rosiglitazone, a specific ligand of peroxisome proliferator-activated receptor $\mathrm{Y}$ (PPARY), has been reported to upregulate DUSP16 expression and inhibit JNK phosphorylation induced by lipopolysaccharides [23]. In contrast, oleanolic acid, a natural compound present in Panax ginseng, has been reported as a potent PPARy agonist [24]. Therefore, NYT might upregulate DUSP16 expression through PPARy activation, 
leading to JNK signal inhibition. However, further investigation is necessary to clarify the detailed mechanism of action of NYT in CSE-induced apoptosis.

The limitations of this study should be acknowledged. The NYT extract powder used in the present study contained numerous compounds that are not absorbed in the body. Therefore, these results might not reflect the actual clinical effects of NYT. To clarify the mechanism of action of NYT in the lungs, it is necessary to evaluate the effect of NYT on cigarette smoke-induced lung injury and apoptosis in mice and humans. Further, we treated cells with NYT for $24 \mathrm{~h}$ prior to CSE exposure. We had initially hypothesized that NYT suppressed CSE-induced apoptosis by modulating the expression levels of several proteins, such as that of anti-oxidant enzymes. For this reason, the cells were treated with NYT for $24 \mathrm{~h}$, whereas the cells were treated with other chemicals for a lesser duration before the CSE exposure [25]. However, we demonstrated that NYT might function as a JNK inhibitor, therefore, NYT treatment for a shorter time before CSE exposure might also inhibit CSE-induced apoptosis.

In conclusion, this study clarified that NYT has a protective effect against CSE-induced apoptosis and IL8 release. In addition, NYT inhibited CSE-induced JNK phosphorylation, but not p38 phosphorylation. These results indicate that NYT possibly functions as a JNK-specific inhibitor, leading to the inhibition of CSE-induced apoptosis and inflammation. Finally, these results suggest that NYT may be a promising therapeutic agent for patients with COPD.

\section{Abbreviations}

COPD, chronic obstructive pulmonary disease; NYT, Ninjinyoeito; CSE, cigarette smoke extract; LDH, lactate dehydrogenase; CCK8, cell counting kit-8; ELISA, Enzyme-Linked Immuno Sorbent Assay; IL-8, interleukin-8; ROS, reactive oxygen species.

\section{Declarations}

\section{All methods were performed in accordance with the relevant guidelines and regulations by including a statement.}

\section{Ethics approval and consent to participate}

Not applicable

\section{Consent for publication}

Not applicable

\section{Availability of data and materials}

The datasets used and/or analysed during the current study available from the corresponding author on reasonable request. 
Competing interests

All authors are employees of Kracie Pharma Ltd.

Funding

Not applicable

Authors' contributions

$\mathrm{KM}, \mathrm{NF}$, and RT contributed to the conception and design of the study. KM conducted all experiments, analyzed the data, and wrote the manuscript. NF and RT have revised the manuscript. All authors have approved the final version of the manuscript for publication.

\section{Acknowledgements}

We would like to thank Editage (www.editage.jp) for English language editing.

\section{References}

1. Sherrill DL, Enright PL, Kaltenborn WT, Lebowitz MD: Predictors of longitudinal change in diffusing capacity over 8 years. Am J Respir Crit Care Med 1999, 160(6):1883-1887.

2. Auerbach $\mathrm{O}$, Hammond EC, Garfinkel L, Benante C: Relation of smoking and age to emphysema. Whole-lung section study. N Engl J Med 1972, 286(16):853-857.

3. Baglole CJ, Bushinsky SM, Garcia TM, Kode A, Rahman I, Sime PJ, Phipps RP: Differential induction of apoptosis by cigarette smoke extract in primary human lung fibroblast strains: implications for emphysema. Am J Physiol Lung Cell Mol Physiol 2006, 291(1):L19-29.

4. Shizu M, Itoh Y, Sunahara R, Chujo S, Hayashi H, Ide Y, Takii T, Koshiko M, Chung SW, Hayakawa $\mathrm{K}$ et al: Cigarette smoke condensate upregulates the gene and protein expression of proinflammatory cytokines in human fibroblast-like synoviocyte line. J Interferon Cytokine Res 2008, 28(8):509-521.

5. Miyano K, Nonaka M, Uzu M, Ohshima K, Uezono Y: Multifunctional Actions of Ninjinyoeito, a Japanese Kampo Medicine: Accumulated Scientific Evidence Based on Experiments With Cells and Animal Models, and Clinical Studies. Front Nutr 2018, 5:93.

6. Kuniaki H, Akihiko T, Tetsuya H, Hatsuko M, Tomoko K, Shin O, Sojiro K, Mayumi Y, Fumihiro Y, Shintaro $S$ et al: Improvement in Frailty in a Patient With Severe Chronic Obstructive Pulmonary Disease After Ninjin'yoeito Therapy: A Case Report. Frontiers in Nutrition 2018, 5:71.

7. Hirai K, Homma T, Matsunaga T, Akimoto K, Yamamoto S, Suganuma H, Kashima A, Sato H, Ebato T, Miyata $Y$ et al: Usefulness of Ninjin'yoeito for Chronic Obstructive Pulmonary Disease Patients with Frailty. J Altern Complement Med 2020, 26(8):750-757. 
8. Miyamoto A, Asai K, Kadotani H, Maruyama N, Kubo H, Okamoto A, Sato K, Yamada K, ljiri N, Watanabe T et al: Ninjin'yoeito Ameliorates Skeletal Muscle Complications in COPD Model Mice by Upregulating Peroxisome Proliferator-Activated Receptor gamma Coactivator-1alpha Expression. Int J Chron Obstruct Pulmon Dis 2020, 15:3063-3077.

9. Takayama F, Egashira T, Yamanaka Y: Protective effect of Ninjin-yoei-to on damage to isolated hepatocytes following transient exposure to tert-butyl hydroperoxide. Jpn J Pharmacol 2001, 85(3):227233.

10. Tanaka K, Sawamura S: Therapeutic effect of a traditional Chinese medicine, ren-shen-yang-rongtang (Japanese name: Ninjin'yoeito) on nitric oxide-mediated lung injury in a mouse infected with murine cytomegalovirus. Int Immunopharmacol 2006, 6(4):678-685.

11. Baglole CJ, Sime PJ, Phipps RP: Cigarette smoke-induced expression of heme oxygenase-1 in human lung fibroblasts is regulated by intracellular glutathione. Am J Physiol Lung Cell Mol Physio/ 2008, 295(4):L624-636.

12. Murata K, Fujita N, Takahashi R, Inui A: Ninjinyoeito Improves Behavioral Abnormalities and Hippocampal Neurogenesis in the Corticosterone Model of Depression. Frontiers in Pharmacology 2018, 9:1216.

13. Murata K, Oyama M, Ogata M, Fujita N, Takahashi R: Oral administration of Jumihaidokuto inhibits UVB-induced skin damage and prostaglandin E2 production in HR-1 hairless mice. $J$ Nat Med 2021, 75(1):142-155.

14. Egashira T, Takayama F, Komatsu Y: Changes of materials that scavenge 1,1-diphenyl-2picrylhydrazyl radicals in plasma by per-oral administration of Kampo medicine, Ninjin-yoei-to in rats. $J$ Pharm Pharmacol 2003, 55(3):367-371.

15. Yue J, Lopez JM: Understanding MAPK Signaling Pathways in Apoptosis. Int J Mol Sci 2020, 21(7).

16. Lee H, Park JR, Kim EJ, Kim WJ, Hong SH, Park SM, Yang SR: Cigarette smoke-mediated oxidative stress induces apoptosis via the MAPKs/STAT1 pathway in mouse lung fibroblasts. Toxicol Lett 2016, 240(1):140-148.

17. Deng X, Xiao L, Lang W, Gao F, Ruvolo P, May WS, Jr.: Novel role for JNK as a stress-activated Bcl2 kinase. J Biol Chem 2001, 276(26):23681-23688.

18. Dhanasekaran DN, Reddy EP: JNK signaling in apoptosis. Oncogene 2008, 27(48):6245-6251.

19. Carnevali S, Petruzzelli S, Longoni B, Vanacore R, Barale R, Cipollini M, Scatena F, Paggiaro P, Celi A, Giuntini C: Cigarette smoke extract induces oxidative stress and apoptosis in human lung fibroblasts. Am J Physiol Lung Cell Mol Physiol 2003, 284(6):L955-963. 
20. Ishii T, Matsuse $\mathrm{T}$, Igarashi $\mathrm{H}$, Masuda M, Teramoto $\mathrm{S}$, Ouchi $\mathrm{Y}$ : Tobacco smoke reduces viability in human lung fibroblasts: protective effect of glutathione S-transferase P1. Am J Physiol Lung Cell Mol Physiol 2001, 280(6):L1189-1195.

21. Masuda K, Shima H, Watanabe M, Kikuchi K: MKP-7, a novel mitogen-activated protein kinase phosphatase, functions as a shuttle protein. J Biol Chem 2001, 276(42):39002-39011.

22. Tanoue T, Yamamoto T, Maeda R, Nishida E: A Novel MAPK phosphatase MKP-7 acts preferentially on JNK/SAPK and p38 alpha and beta MAPKs. J Biol Chem 2001, 276(28):26629-26639.

23. Hwang JS, Ham SA, Yoo T, Lee WJ, Paek KS, Kim JH, Lee CH, Seo HG: Upregulation of MKP-7 in response to rosiglitazone treatment ameliorates lipopolysaccharide-induced destabilization of SIRT1 by inactivating JNK. Pharmacol Res 2016, 114:47-55.

24. Ferguson HE, Thatcher TH, Olsen KC, Garcia-Bates TM, Baglole CJ, Kottmann RM, Strong ER, Phipps RP, Sime PJ: Peroxisome proliferator-activated receptor-gamma ligands induce heme oxygenase-1 in lung fibroblasts by a PPARgamma-independent, glutathione-dependent mechanism. Am J Physiol Lung Cell Mol Physiol 2009, 297(5):L912-919.

25. Chen M, Yang T, Meng X, Sun T: Azithromycin attenuates cigarette smoke extract-induced oxidative stress injury in human alveolar epithelial cells. Mol Med Rep 2015, 11(5):3414-3422.

\section{Tables}

Due to technical limitations, table 1 is only available as a download in the Supplemental Files section.

\section{Figures}


(A)

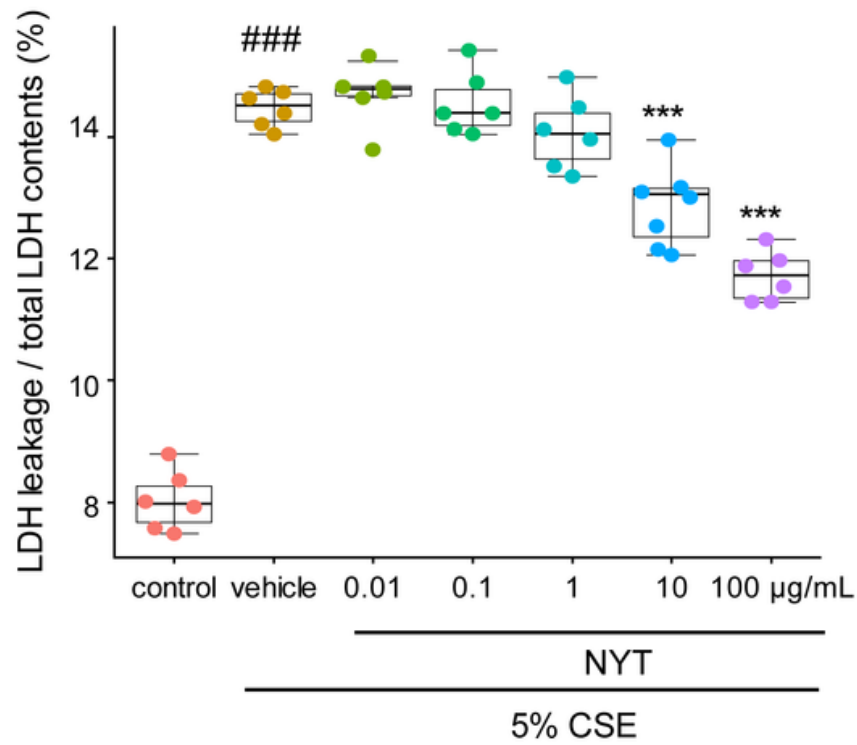

(B)

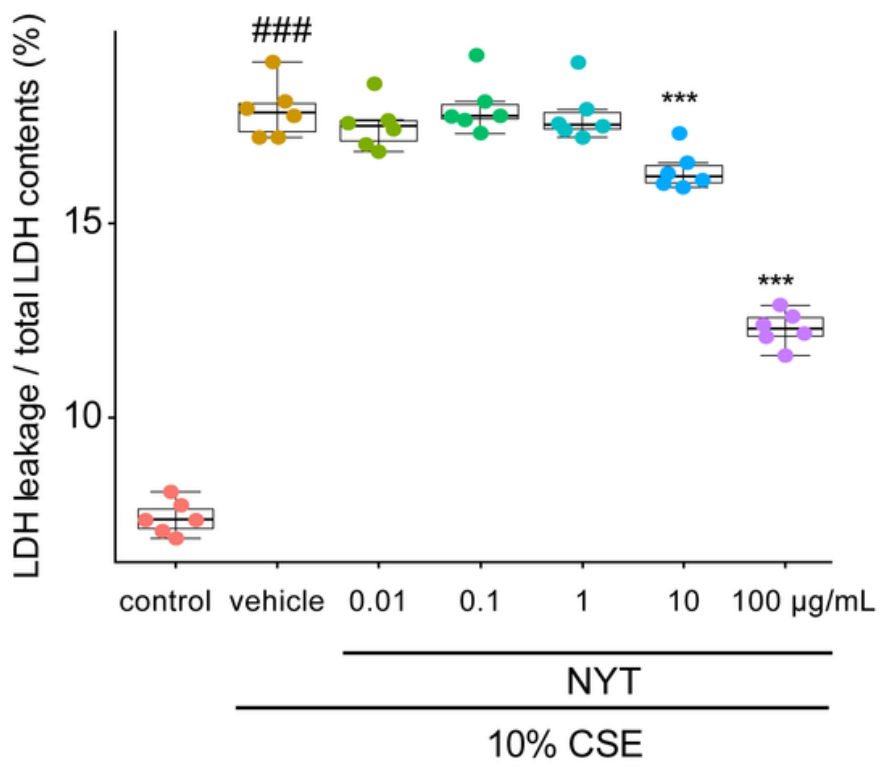

(C)

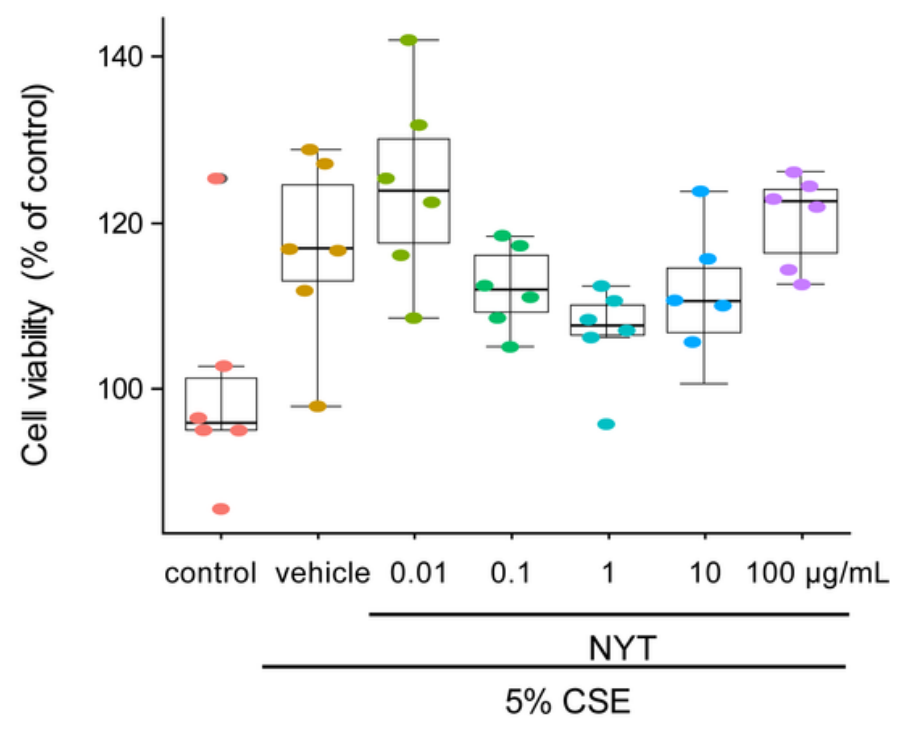

(D)

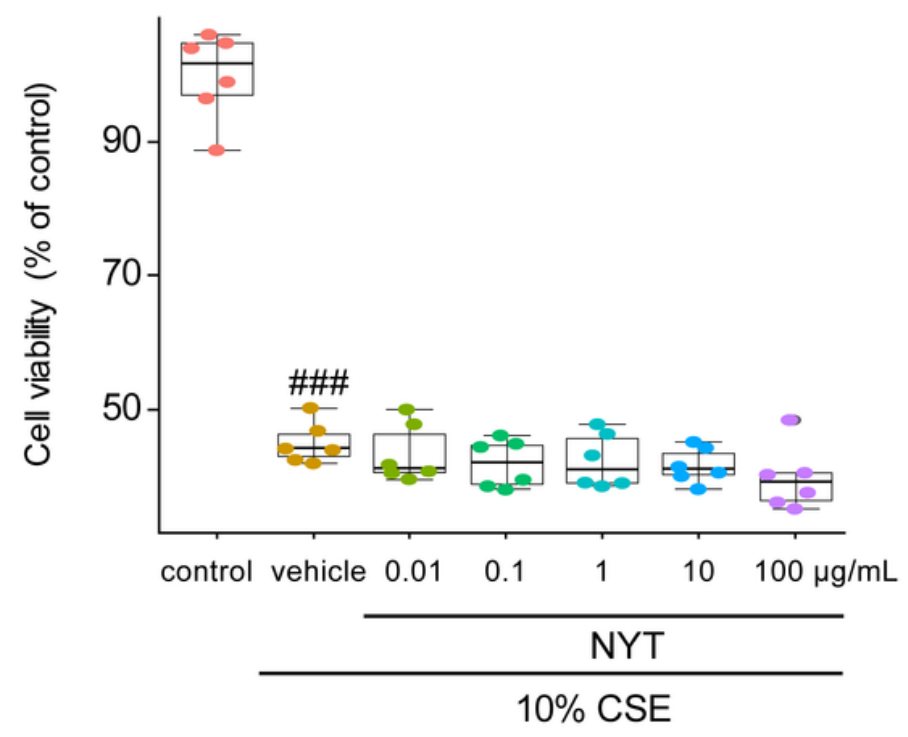

\section{Figure 1}

NYT ameliorated CSE-induced LDH leakage The effect of NYT on CSE-induced cytotoxicity was evaluated using an LDH assay kit or a CCK-8 assay kit. The cells were treated with NYT for $24 \mathrm{~h}$ prior to exposure to $5 \%(A, C)$ or $10 \%$ CSE (B, D). Twenty-four hours after CSE exposure, cytotoxicity was measured by LDH leakage (A, B) and cell viability by the CCK-8 assay (C, D). \#\#\#p $<0.001$ vs. control group; ${ }^{* \star p}<<0.01$, $* \star \star p$ $<0.001$ vs. vehicle-treatment group; Tukey's test; $n=6$. CSE: cigarette smoke extract; NYT: Ninjinyoeito 


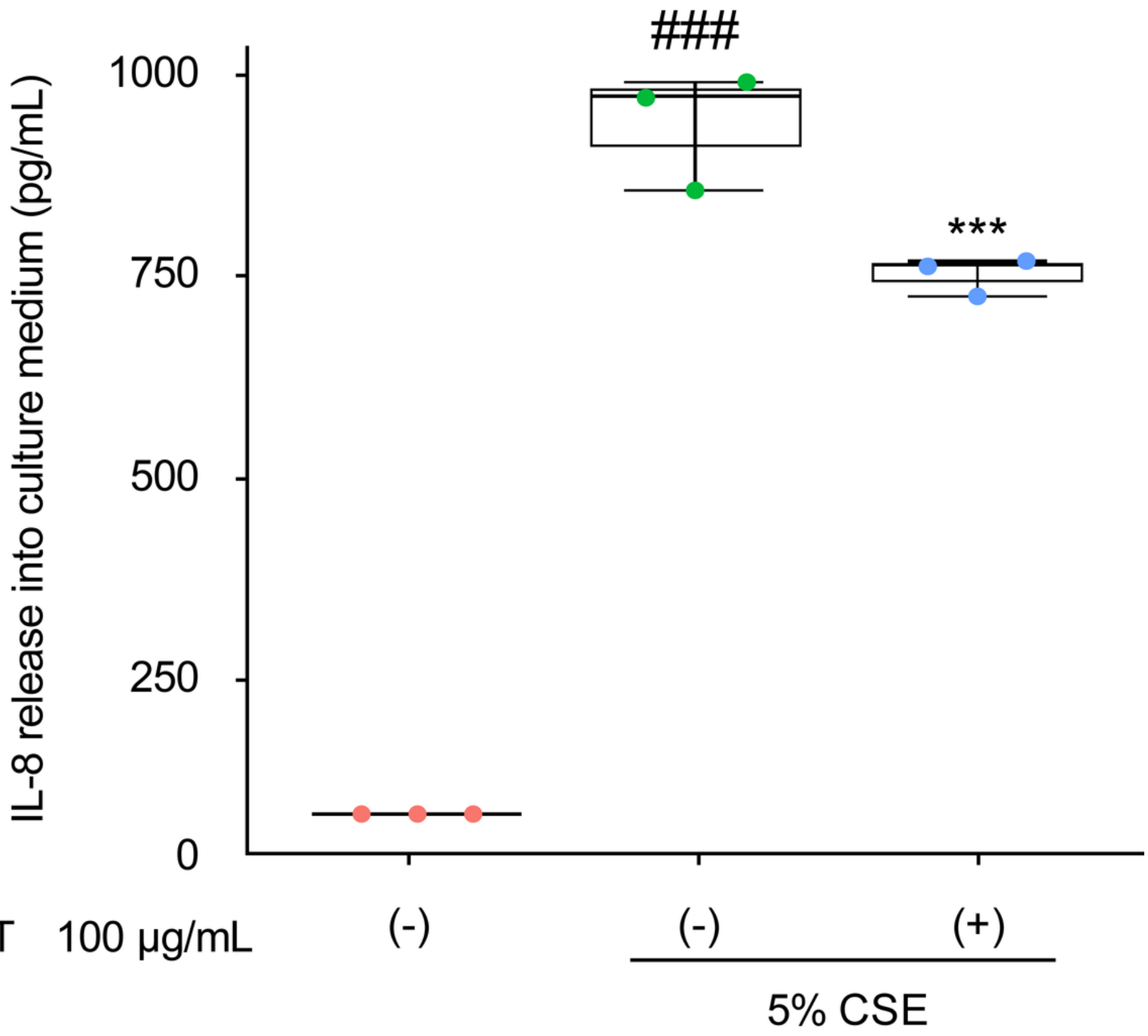

Figure 2

NYT ameliorated CSE-induced IL-8 release The effect of NYT on CSE-induced IL-8 release was evaluated. The amount of IL-8 in the cell culture medium at $24 \mathrm{~h}$ after exposure to $5 \%$ CSE was measured using an IL-8 ELISA assay kit. \#\#\#p < 0.001 vs. control group; ${ }^{* \star} p<0.001$ vs. vehicle-treatment group; Tukey's test; $\mathrm{n}=3$. CSE: cigarette smoke extract; NYT: Ninjinyoeito 
(A)

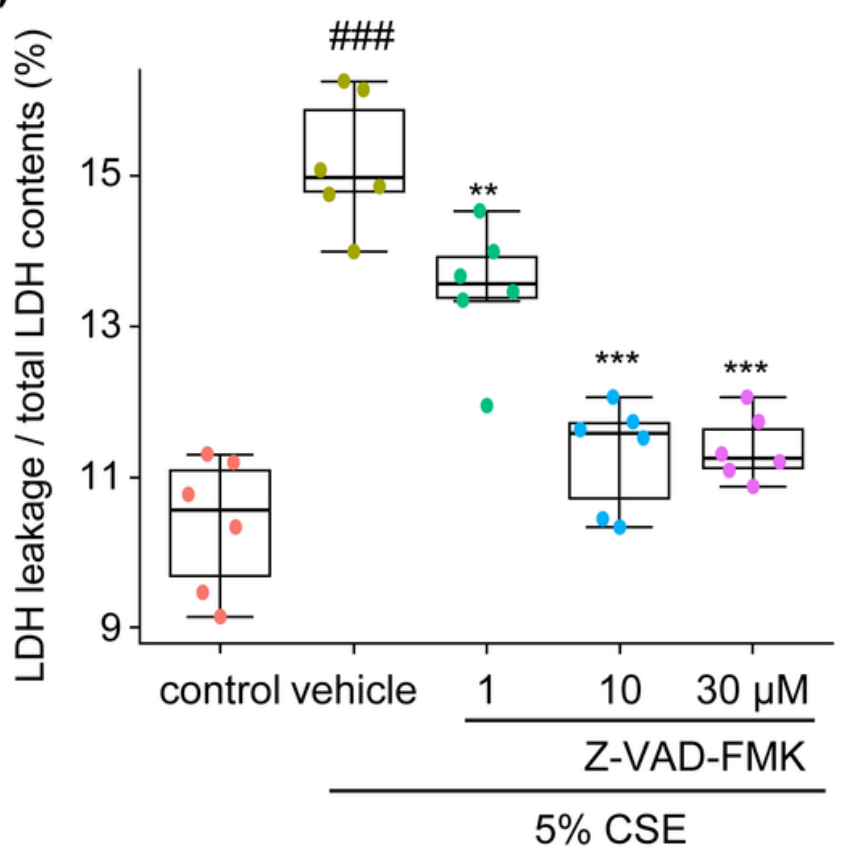

(B)

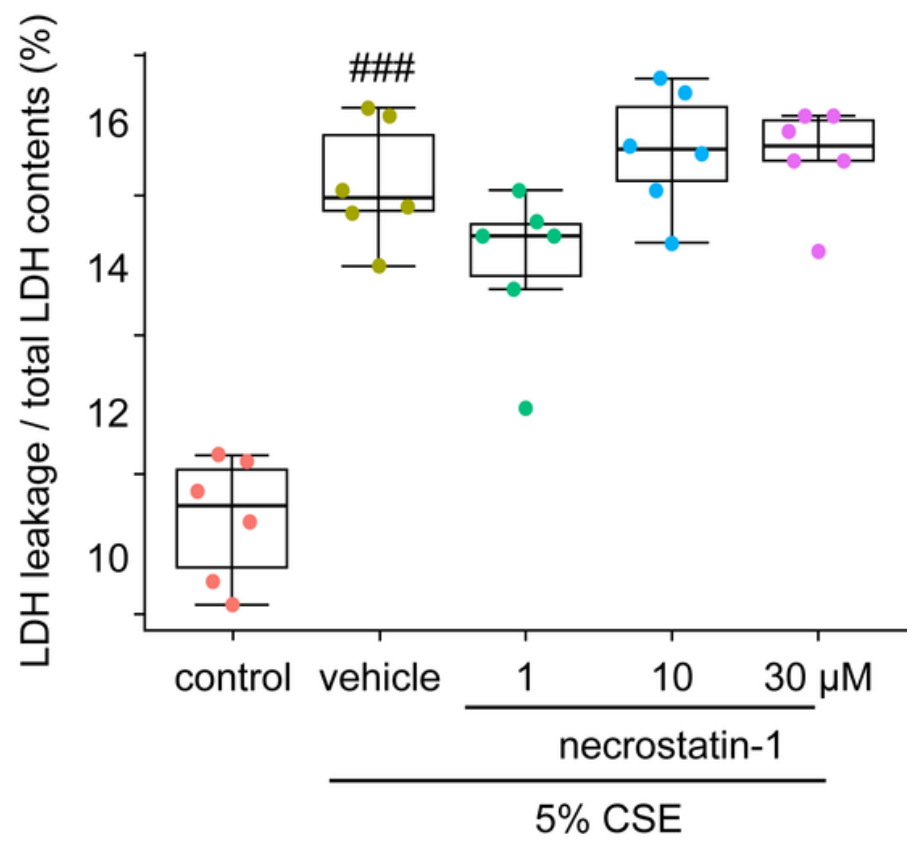

Figure 3

CSE exposure induced caspase-dependent apoptosis. The effects of specific inhibitors of apoptosis and necrosis were evaluated using an LDH assay kit. Two hours before exposure to $5 \%$ CSE, the cells were treated with either Z-VAD-FMK (A) or necrostatin-1 (B) to inhibit apoptosis and necrosis, respectively. The cytotoxicity of LDH leakage was evaluated $24 \mathrm{~h}$ after CSE exposure. \#\#\#p < $0.001 \mathrm{vs.} \mathrm{control} \mathrm{group;} * \star \star p$ $<0.001$ vs. vehicle-treatment group; Tukey's t-test; $n=6$. CSE: cigarette smoke extract; NYT: Ninjinyoeito 
(A)

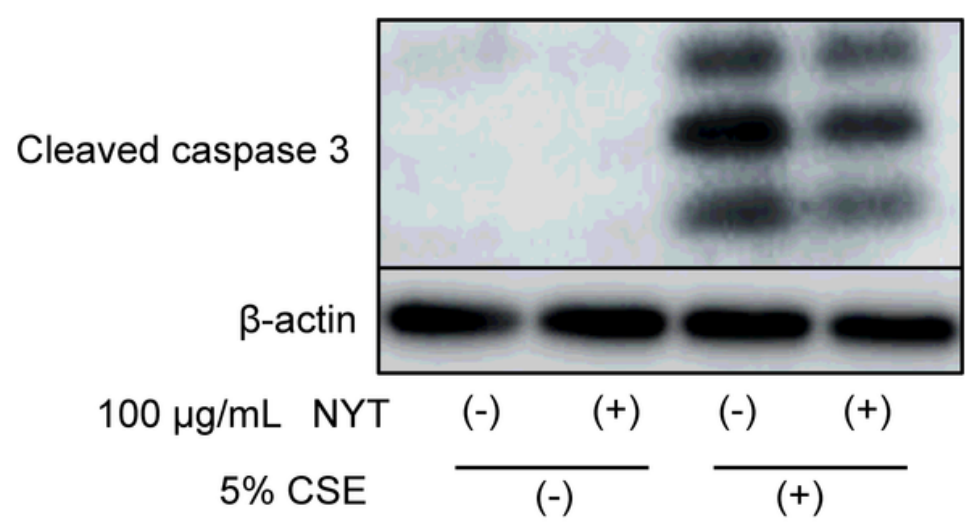

(C)

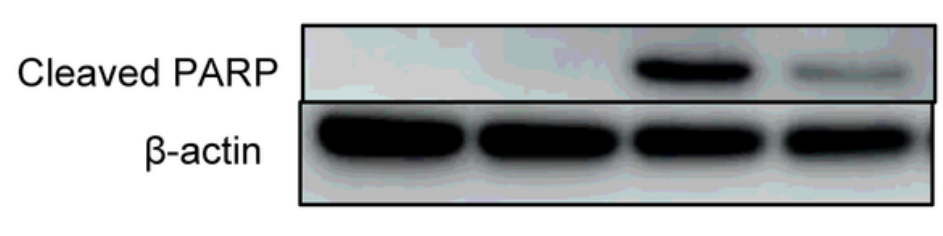
$100 \mu \mathrm{g} / \mathrm{mL}$ NYT
$(-) \quad(+) \quad(-)$ $5 \%$ CSE
$(-)$

(B)

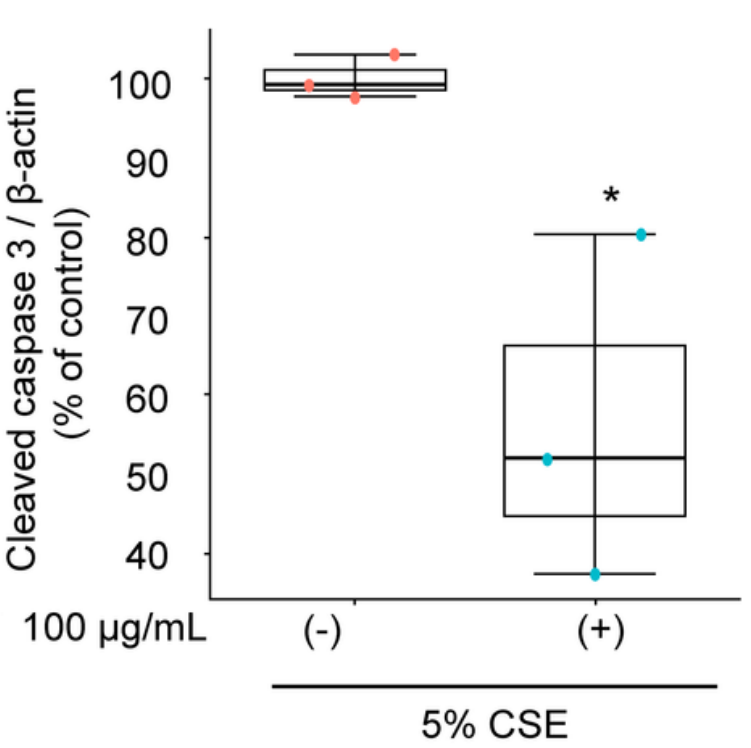

(D)

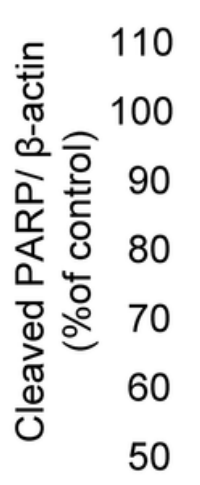

NYT $100 \mu \mathrm{g} / \mathrm{mL}$

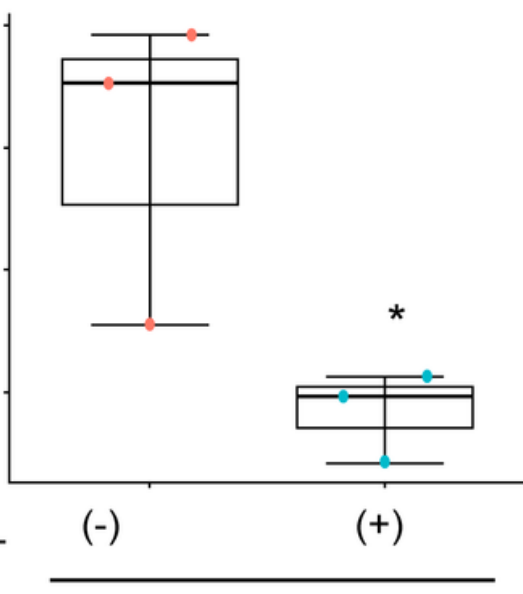

5\% CSE

Figure 4

NYT inhibited CSE-induced cleavage of caspase 3 and PARP. The effect of NYT on the expression levels of cleaved caspase 3 and PARP was evaluated by western blotting analysis (A-D). Twenty-four hours after CSE exposure, the cells were collected, and western blotting analysis was performed to evaluate the expression levels of cleaved caspase 3 (B) and cleaved PARP (D). The full-length blot images were presented in Supplementary figure 1. \#\#\#p<0.001 vs. control group; ${ }^{\star \star \star} p<0.001$ vs. vehicle-treatment group; Student's t-test; $n=3$. CSE: cigarette smoke extract; NYT: Ninjinyoeito 
(A)

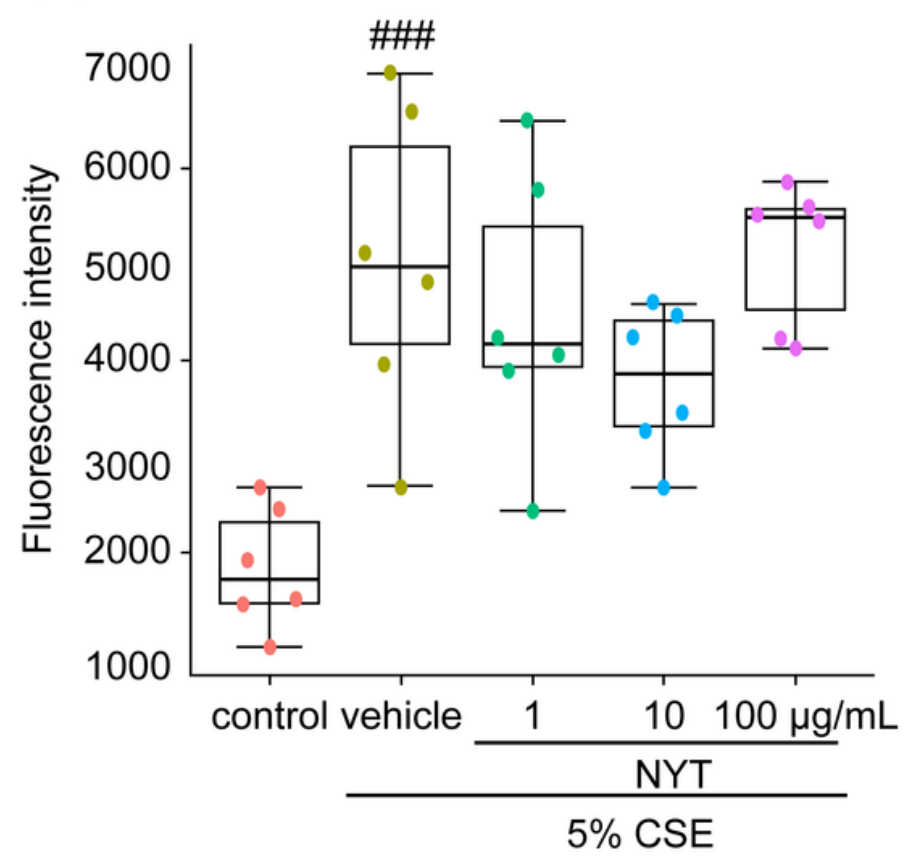

(B)

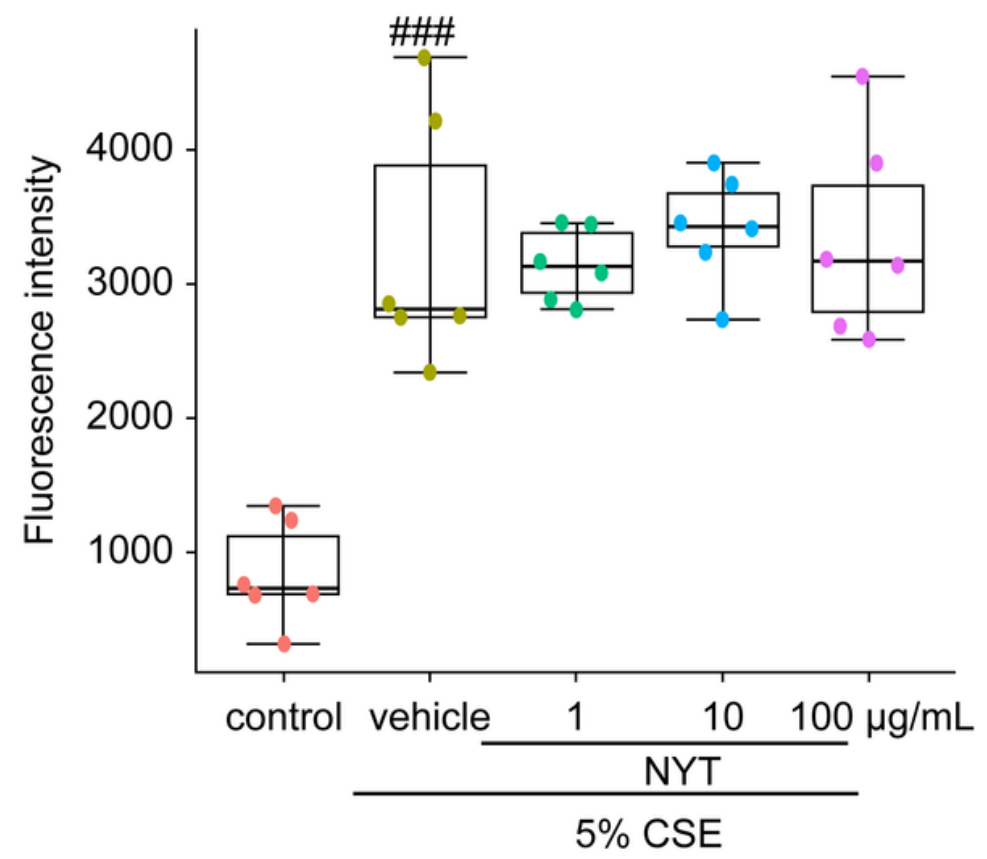

Figure 5

NYT did not change the CSE-induced ROS production. The effect of NYT on CSE-induced ROS production was also evaluated. Intracellular ROS generation was measured using CellROX green reagent at $8 \mathrm{~h}(\mathrm{~A})$ or 24 h (B) after CSE exposure. \#\#\#p < 0.001 vs. control group; Tukey's test. CSE: cigarette smoke extract; NYT: Ninjinyoeito

(B)

\section{(A)}

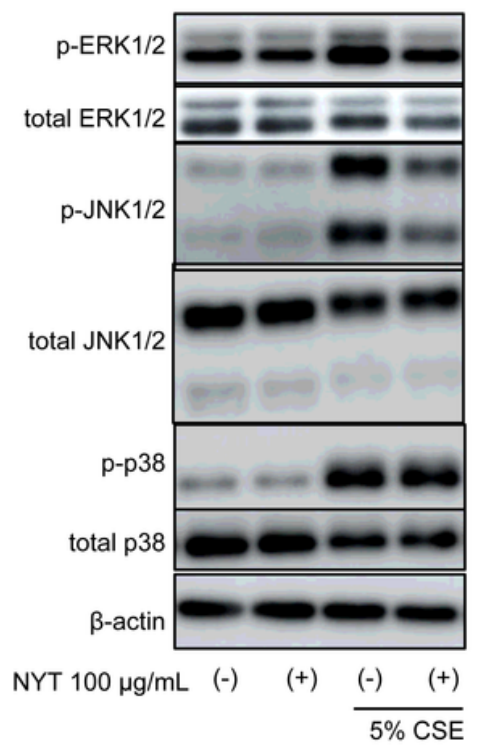

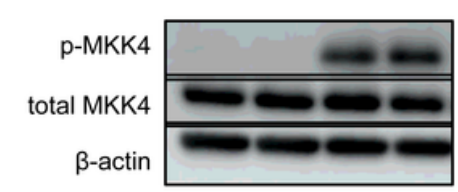

NYT $100 \mu \mathrm{g} / \mathrm{mL} \quad(-) \quad(+) \frac{(-) \quad(+)}{5 \% \mathrm{CSE}}$

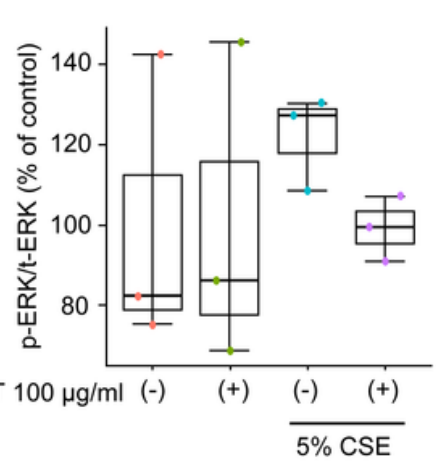

(D)

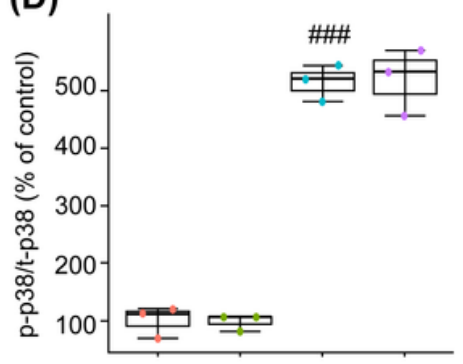

(C)

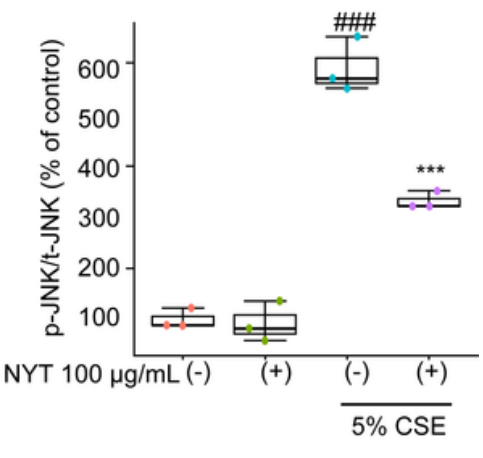

(E)

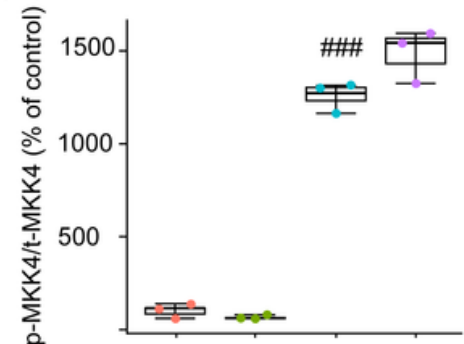

$\mathrm{NYT} 100 \mu \mathrm{g} / \mathrm{mL} \quad\left(_{-} \quad\left(^{+}\right) \frac{(-) \quad(+)}{5 \% \text { CSE }}\right.$ NYT $100 \mu \mathrm{g} / \mathrm{mL}\left(^{-}\right) \quad(+) \frac{(-) \quad(+)}{5 \% \text { CSE }}$

Figure 6 
NYT inhibited CSE-induced JNK signal activation The effect of NYT on the MAPK signaling pathway was evaluated (A). Twenty-four hours after CSE exposure, the cells were collected, and western blotting analysis was performed to evaluate the phosphorylation levels of ERK1/2 (B), JNK (C), p38 (D), and MKK4 (E). The full-length blot images were presented in Supplementary figure 2. \#\#\#p $<0.001 \mathrm{vs}$. control group; ${ }^{* \star} p<0.001$ vs. vehicle-treatment group; Tukey's t-test; $n=3$. CSE: cigarette smoke extract; NYT: Ninjinyoeito

\section{Supplementary Files}

This is a list of supplementary files associated with this preprint. Click to download.

- table1.xlsx

- supplementaryfigure1.tif

- supplementaryfigure2.tif

- SupplementaryFigurelegend.docx 